(1985) Pferdeheilkunde 1, 255-259

\section{Röntgenologie des Karpalgelenks}

\section{P. N. Burguez}

Beaufort Cottage Stables, Newmarket, Suffolk, England

\section{Einleitung}

Die Komplexität des Karpalgelenks, das aus drei Hauptgelenken, sieben oder acht Karpalknochen und fünf angrenzenden Knochen besteht, von denen jeder mit einem oder mehreren Bestandteilen des Karpus gelenkig in Verbindung steht, macht für eine eindeutige Beurteilung Röntgenaufnahmen von hoher Qualität erforderlich. Die Interpretation ist unter Umständen äußerst schwierig, da das Verhältnis zwischen röntgenologischen Veränderungen und klinisch auftretenden Störungen unterschiedlich ist. Sie muß daher unter Berücksichtigung der Befunde aus einer gründlichen klinischen Untersuchung geschehen.

\section{Röntgentechnik}

Es sind fünf Standardprojektionen möglich. Sie bestehen in den seitlichen Aufnahmen bei gebeugtem und gestrecktem Gelenk, der kraniokaudalen (anterioposterioren) mediolateralen (APMLO) und kraniokaudalen lateromedialen (APLMO) Schrägansicht im $45^{\circ}$-Winkel und dem Bild mit kraniokaudalem (AP) Strahlengang. Smallwood und Shivelly (1979) beschrieben Beispiele dieser Projektionen bei normalen Pferden. Zusätzlich sind manchmal Darstellungen der kranialen Begrenzung der proximalen und der distalen Karpalknochenreihe im tangentialen Strahlengang hilfreich. Häufig sind die Veränderungen am einfachsten auf einer oder zwei der Standardprojektionen zu sehen. Wo dies zutrifft, geht aus dem Text hervor.

\section{Beurteilung}

Die Erscheinungen auf der Röntgenaufnahme stellen so oft kein wahres Bild des pathologischen Zustands im Gelenk dar, der bei einer nachfolgenden Operation oder Autopsie festgestellt wird, daß man begonnen hat, mit Hilfe einer Analyse der Bestandteile der Gelenkflüssigkeit (Tew und Hotchkiss, 1981) und der Arthroskopie (McIllwraith und Fessler, 1978) die Bedeutung und Prognose röntgenologischer Veränderungen zu bewerten.

Diese Veröffentlichung möchte Erscheinungsbilder ohne klinische Relevanz, solche mit unterschiedlicher und diejenigen mit tatsächlicher klinischer Bedeutung beschreiben.

\section{Röntgenbefunde obne Bedeutung für die Klinik}

Das Bild, das eine AP-Ansicht von der medialen Fläche der distalen Radiusmetaphyse liefert, wird manchmal zu Un- recht als sekundäre Knochenzubildung infolge eines Traumas diagnostiziert (Adams, 1974). Myers (1965) sprach von einem unvollständigen Schluß der Verschmelzungslinie zwischen dem ursprünglichen distalen Ulnaende (Processus styloideus) und der distalen Radiusepiphyse. Dieser Autor gab an, daß die Vereinigung bei $40 \%$ der zwei- bis vierjährigen Pferde und bei $30 \%$ der über 4 Jahre alten Pferde nicht abgeschlossen ist (Abb. 1). Auch vom Auftreten einer beidseitigen vollständigen Ulna bei einer dreijährigen Stute berichtete derselbe Autor. Man hat sie auch in unserer Praxis beobachtet und, in einer Aufeinanderfolge von Fällen, zeigten 10 von 29 zweijährigen Vollblütern bilateral eine vollständige Ulna (Abb. 2). Adams (1974) beschrieb eine vertikale Aufhellungslinie, die gelegentlich im Os carpi accessorium sichtbar war (Abb. 2). Man sollte sie nicht mit einer Fraktur verwechseln. Ein kleiner Prozentsatz von Pferden besitzt ein separates Os carpale primum medial und kaudal vom Os carpale secundum. Adams (1974) wies auch auf ein "nicht-verschmolzenes Os carpale quintum“ an der Kaudolateralfläche des Karpus hin. Diese Knochen können von frakturierten Fragmenten durch ihre Position und ihr glatt umrundetes Erscheinungsbild abgegrenzt werden.

Als anatomisch normale Tatsache ist der Grad der Rotation des Os carpi accessorium beim Abbeugen des Karpalgelenks interessant (J. Hickman, 1982; persönliche Mitteilung). Man kann ihn durch Vergleich der lateralen Ansicht von belasteter und gebeugter Gliedmaße bestimmen.

\section{Röntgenologische Veränderungen von unterschiedlicher klinischer Relevanz}

Die Hyperextension des Karpus darf als physiologische anatomische Variante angesehen werden, außer, wenn sie die Ursache pathologischer Erscheinungen klinisch und röntgenologisch darstellt. Deshalb rechnet man sie zu dieser Gruppe. Manning und St. Clair (1972) berichteten von einer „Abwärtswinkelung des Os carpi accessorium, Periostitis an der lateralen und kaudalen Oberfläche des distalen Radiusteils, Verlagerung des proximalen Radiusendes nach kranial und alterierte Gelenkkapselbereiche an der Dorsalfläche des Radiokarpalgelenks als den pathologischen Erscheinungen, die öfter im Zusammenhang mit der Hyperextension des Karpus auftreten. Sie gaben an, daß 80 Prozent von 86 Pferden, die wegen unterschiedlicher Frakturen im Karpalgelenk untersucht wurden, röntgenologisch eine oder mehrere dieser Veränderungen zeigten, und erstellten die These, die Hyperextension des Karpus könne die Folge von Alterationen am Stützapparat des Os carpi accessorium sein.

Periostreaktionen an der Kranialfläche des Os carpi radiale und Os carpale tertium sind ein häufiger Befund. Normalerweise stehen sie in Verbindung mit dem Abreißen der in diesem Bereich ansetzenden Gelenkkapsel, können aber in einigen Fällen auch durch ein perkutanes Trauma verursacht sein. Man betrachtet die Veränderung nicht als klinisch relevant, solange nicht Erscheinungen wie Synovitis oder röntgenologisch sichtbare osteochondrale Fragmente sie begleiten oder das Gelenk beteiligt ist. Wichtig ist, das Ausmaß der periostalen Reaktion einzuschätzen (Abb. 4) 


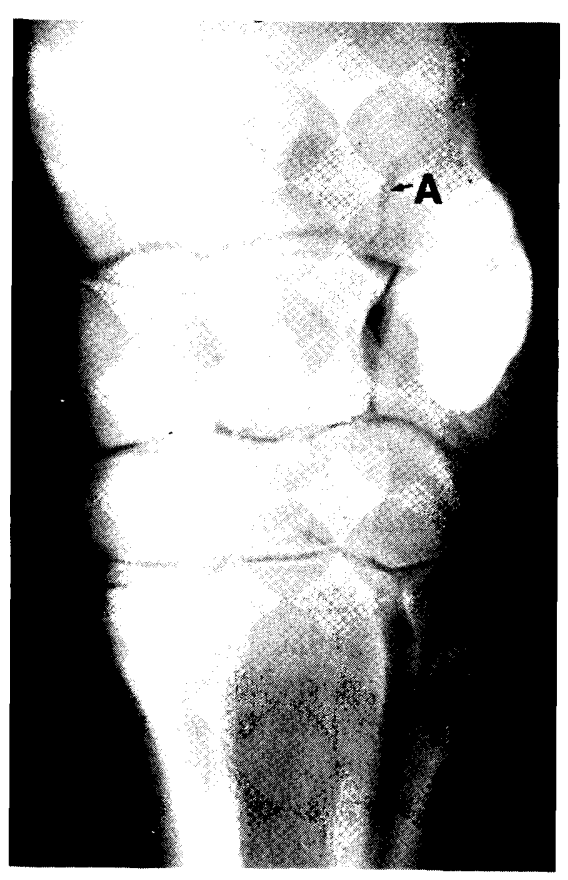

Abb. 1: APLMO-Ansicht bei einem zweijährigen Vollblüter, die den unvollständigen Schluß der Verschmelzungslinie zwischen distalem Ulnaende (Processus styloideus) und distaler Radiusepiphyse zeigt (A).
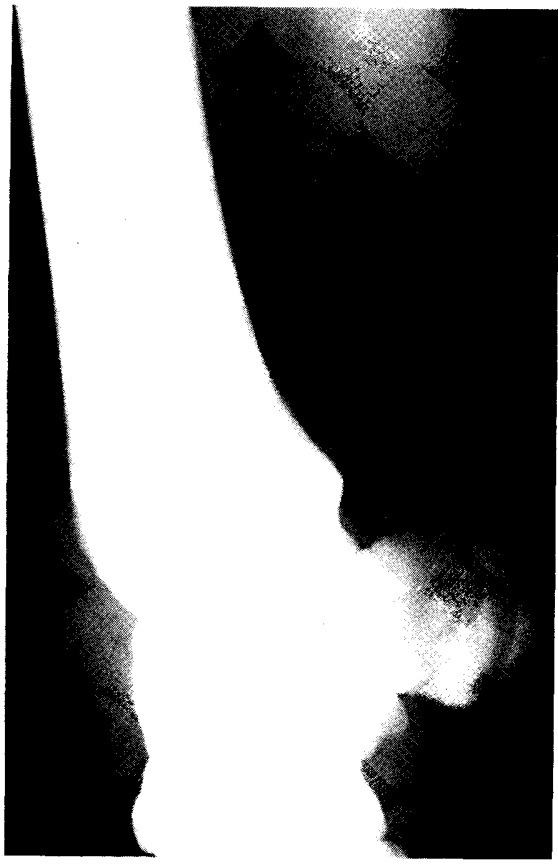

Abb. 2: Laterale Aufnahme einer zweijährigen Vollblutstute, auf der eine Aufhellungslinie (nicht zu verwechseln mit einer Fraktur) im Os carpi accessorium (Pfeile) und eine vollständige Uina $(A)$ zu sehen sind.

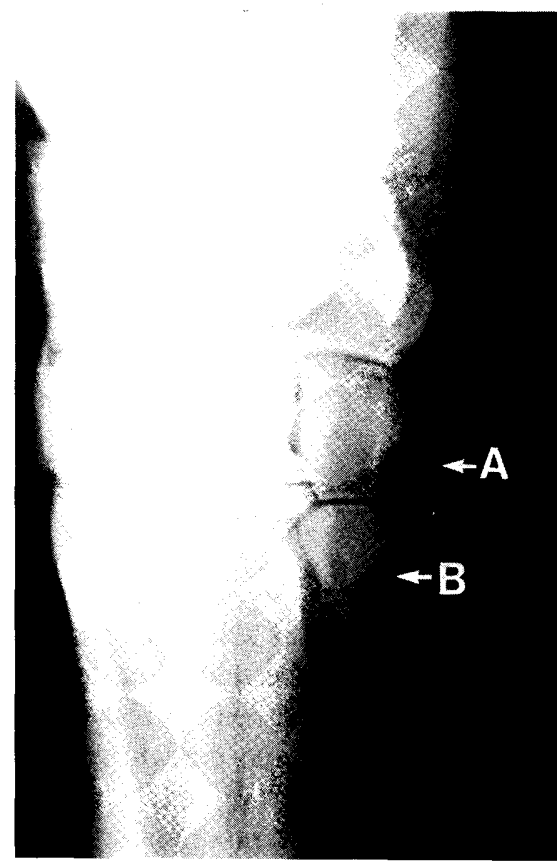

Abb. 3: APLMO-Ansicht bei einem erwachsenen Pferd, in der man einen fünften Karpalknochen $(A)$ und eine Knochenzyste im lateralen Griffelbeinköpfchen (B) sieht. und, ob sie im Gelenk abläuft oder nicht. Abb. 3 zeigt eine Knochenzyste im medialen Griffelbeinköpfchen. Dieses Zustandsbild wurde in der Equine Research Station zweimal in den vergangenen fünf Jahren gesehen. Die Infiltration eines Lokalanästhetikums über dem betroffenen Be-
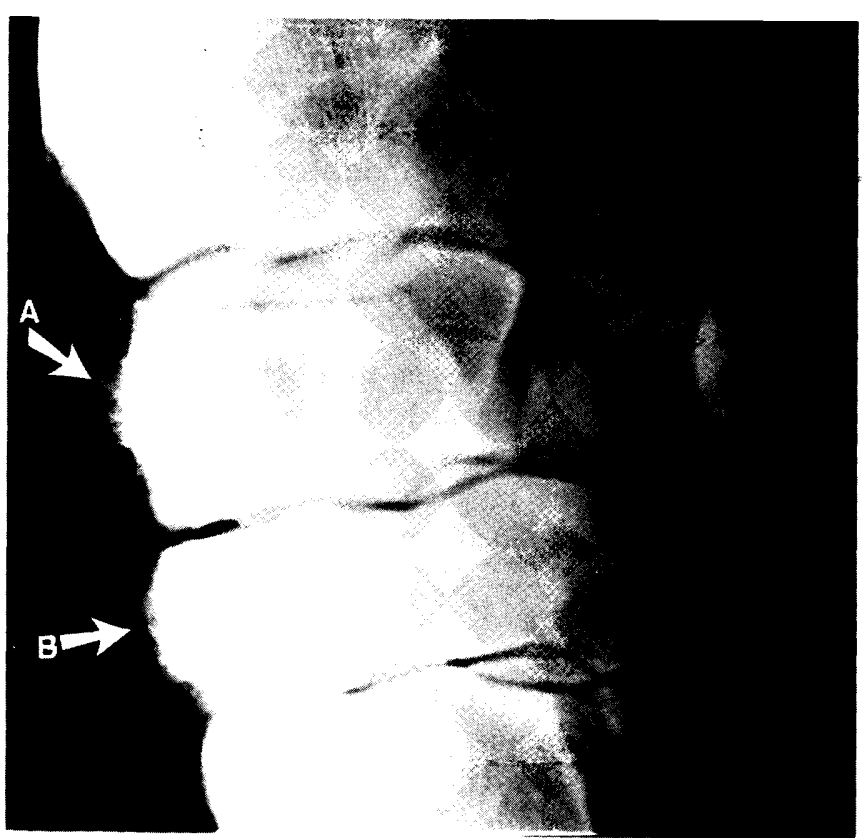

Abb. 4: APLMO-Ansicht bei einem dreijährigen Vollblüter, die eine Periostreaktion an der Kranialfläche des Os carpi radiale (A) und des Os carpale tertium zeigt. Man beachte das "dornförmige" Erscheinungsbild, das darauf hinweist, daß es sich um eine akute Alteration handelt und die Knochenzubildung außerhalb des Gelenks ist. reich führte zur Schmerzfreiheit, und ein sechs Monate später aufgenommenes Röntgenbild zeigte eine Größenabnahme sowie eine Zunahme der Knochengewebsdichte an der Stelle der Läsion. Zu dieser Zeit war das Pferd wieder gesund. (L. B. Jeffcott, 1982; persönliche Mitteilung).

\section{Röntgenologische Erscheinungsbilder} mit erwiesener klinischer Bedeutung

Degenerative joint disease

(DJD, Arthropathia deformans).

Die Läsionen sind hauptsächlich an der kraniomedialen Fläche des Interkarpalgelenks festzustellen, gelegentlich auch am Radiokarpalgelenk und sehr selten am Karpometakarpalgelenk. Das Stadium der fortschreitenden Gelenkserkrankung bestimmt wesentlich die sichtbaren röntgenologischen Veränderungen. Auf Grund der relativ konstanten Lage der vom Degenerationsprozeß betroffenen Knochenstelle ist die APLMO-Ansicht sehr wichtig. Anfangs treten unter Umständen keine erkennbaren röntgenologischen Erscheinungen auf, und trotzdem können klinisch Anzeichen einer Erkrankung vorhanden sein.

Als erstes ist auf dem Röntgenbild eine verringerte Dichte des Knochengewebes an der kraniomedialen Gelenkfläche bemerkbar: Die Erkennung dieser frühesten Veränderung erfordert Sorgfalt - die Stelle mit verminderter Dichte in dem Interkarpalgelenk der Abb. 5 ist nicht als klinisch relevant anzusehen, da sie üblicherweise in der APLMO-Ansicht auftritt, wenn der Zentralstrahl zu weit kranial gerichtet ist (worauf die Projektion des Os carpi accessorium hindeutet). Die darauffolgende röntgenologische Veränderung besteht in der Ausbildung von Osteophyten oder 


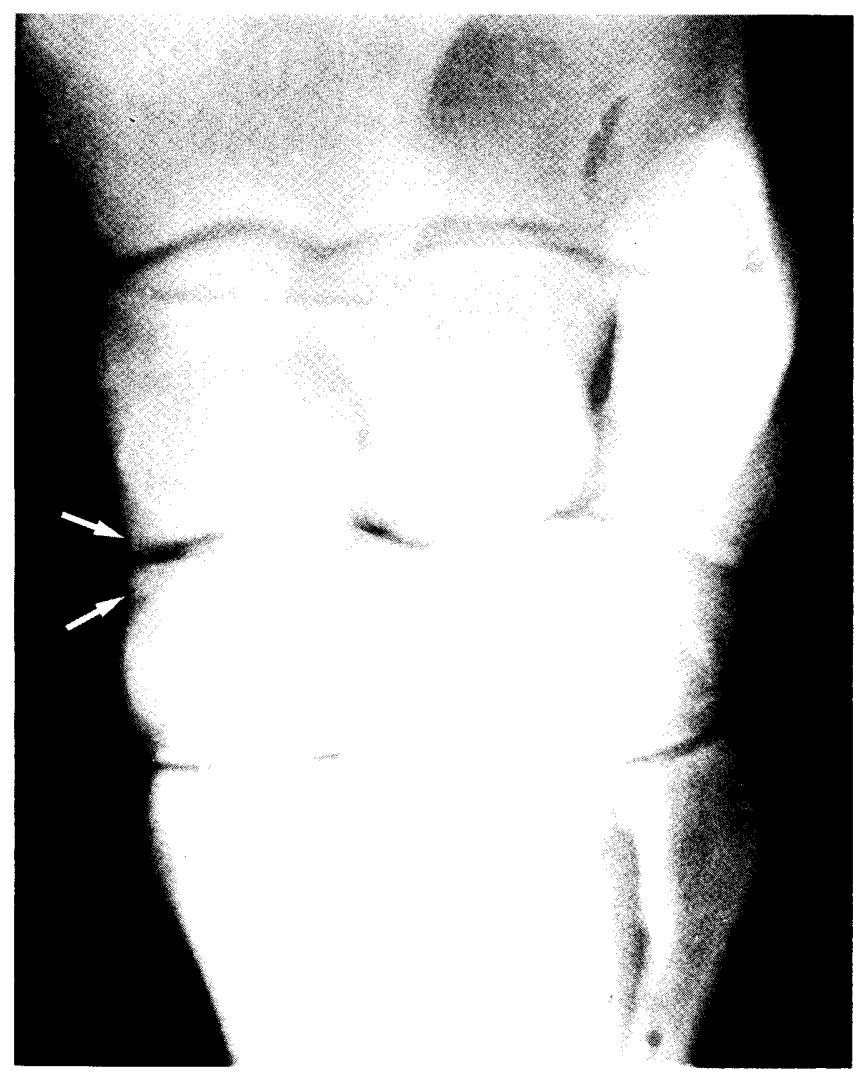

Abb. 5: APLMO-Ansicht, bei der der Röntgenzentralstrahl zu weit kranial gerichtet und in welcher auf der kraniomedialen Fläche ein Bereich mit verminderter Dichte (Pfeile) zu sehen ist. Man vergleiche die Lage des Os carpi accessorium mit der in Abb. 4.

„Exostosen“, die entweder groß sein und im Vergleich zum Knochen, von dem sie ausgehen, eine geringere Dichte aufweisen können (Abb.6), oder ganz klein und aus ohne sichtbar in seiner Dichte reduziertem Gewebe. Dieses veränderte röntgenologische Erscheinungsbild wird als sekundäre Umformung des Osteophyten angesehen. Ein primär umgeformter Osteophyt führt zur Veränderung der Gestalt des gesamten betroffenen Karpalknochens, was nach Ansicht des Autors eine Folge der individuell in unterschiedlicher Stärke und verschiedenen Richtungen einwirkenden Kräfte bei der Belastung ist. Man muß aber betonen, daß manche Pferde auf dem Röntgenbild Veränderungen in forgeschrittenen Stadien zeigen, aber klinisch ohne Anzeichen einer Erkrankung sind.

\section{Chip-Frakturen}

Chip-Frakturen können in frakturierte Osteophyten und Abrißfrakturen unterteilt werden. Park, Morgan und O'Brien (1970) fanden, daß man diese Frakturen am häufigsten in der lateralen Ansicht bei gebeugter Gliedmaße diagnostizieren konnte. Sie vertraten die Meinung, daß mit Hilfe der Aufnahme in diesem Strahlengang auch eine bessere Beurteilung der Beweglichkeit des Frakturfragments möglich sei, indem man sie mit dem Röntgenbild der gestreckten Gliedmaße in lateraler Ansicht (Abb. 7 und 8) vergleicht. Auch gelangten sie zur Überzeugung, daß der AP-Strahlengang die wenigsten Frakturen sichtbar werden ließ, bei der Bewertung des Gelenkspalts jedoch gute Dienste leistete.

Park et al. (1970) und Thrall, Lebel und O'Brien (1971) kamen zu dem Schluß, daß in den meisten Fällen das Os carpi radiale von Chip-Frakturen betroffen ist, und am häufigsten am distalen kraniomedialen Rand. Ein gutes Untersuchungsprinzip ist, Aufnahmen von lateral bei APLMOStrahlengang im Winkel von $30^{\circ}$ und $45^{\circ}$ zu machen, um die Veränderung im Hinblick auf eine exakte Diagnose und/oder chirurgische Korrektur klar herauszustellen (Abb. 9 und 10). Das Os carpi accessorium ist als Richtungsindikator wichtig. Zum Beispiel zeigt Abb. 9 mehr vom Os carpi accessorium als Abb. 10 und ist daher die Schrägansicht von lateral im $30^{\circ}$-Winkel.

Die röntgenologischen Erscheinungen geben möglicherweise kein zutreffendes Bild vom aktuellen Stand des am
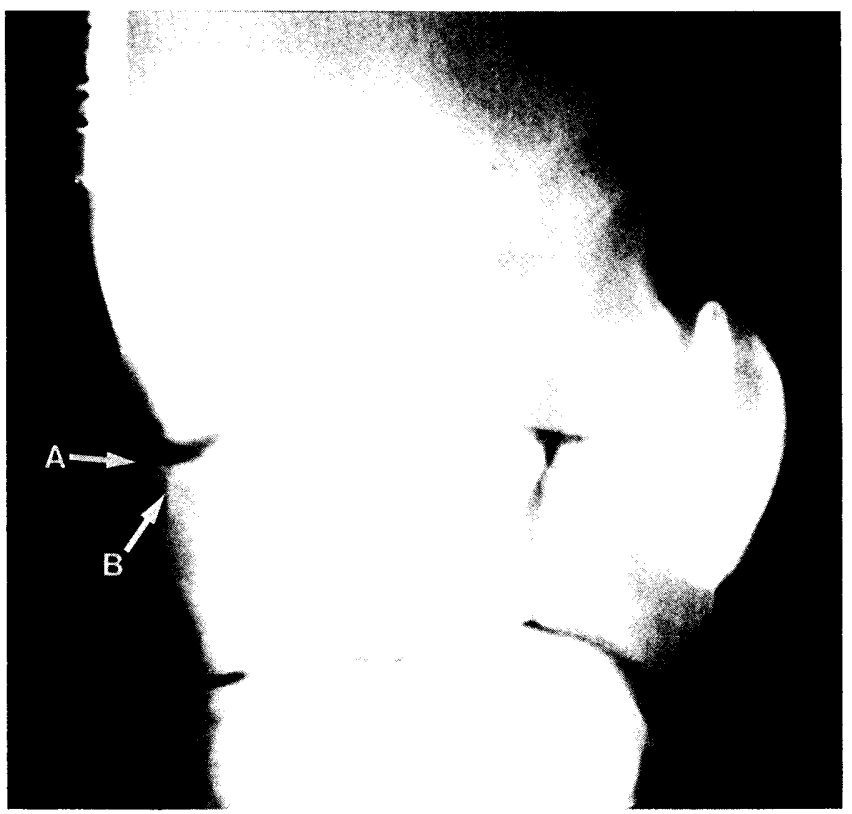

4 Abb. 6: APLMO-Aufnahme einer zweijährigen Vollblutstute, auf der man einen großen Osteophyten an der proximalen kraniomedialen Begrenzung des Os carpi radiale $(A)$ sieht. Man beachte die Größe, die verringerte Dichte und die mögliche Frakturlinie (B).

Abb. 7: Laterale Ansicht der beasteten Gliedmaße eines Vieriährigen, die eine Periostreaktion entlang der kranialen Begrenzung des Os carpi radiale (A) und eine Chip-Fraktur zeigt.

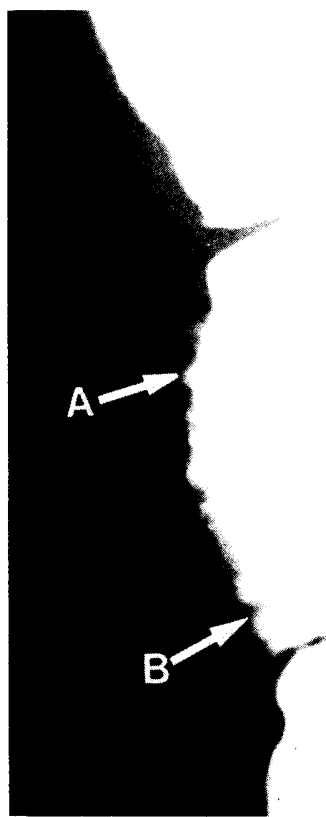



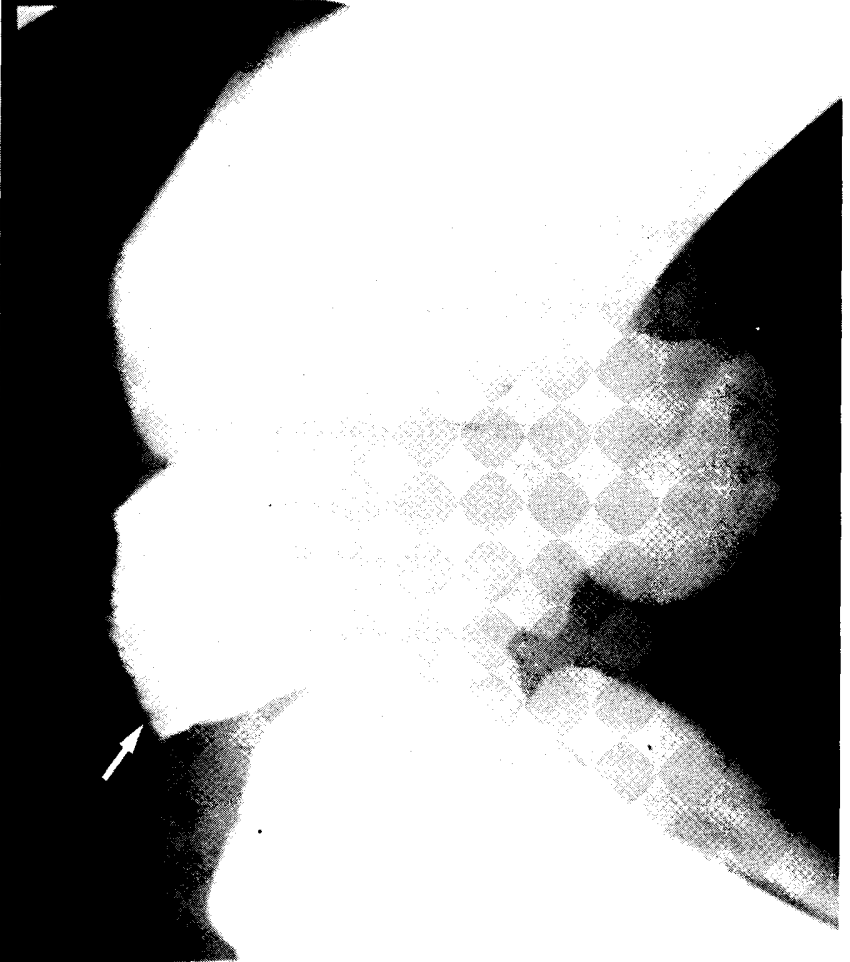

Abb. 8: Laterale Ansicht bei gebeugter Gliedmaße desselben Pferds wie in Abb. 7, in der zu sehen ist, daß die Chip-Fraktur unbeweglich ist.

Gelenk ablaufenden pathologischen Prozesses, weil Knorpelveränderungen sich nicht im Röntgenbild zeigen. Ein Dreijähriger „ging nicht richtig vom Fleck“, wie sein Trainer berichtete, und die Röntgenaufnahmen zeigten eine subchondrale Knochenaussparung am distalen kranialen Rand des Os carpi radiale. Der Trainer bat darum, das Training planmäßig fortzusetzen, und 10 Tage später trat bei dem Pferd eine akute Lahmheit auf. Zu diesem Zeitpunkt aufgenommene Röntgenbilder brachten eine große Chip-
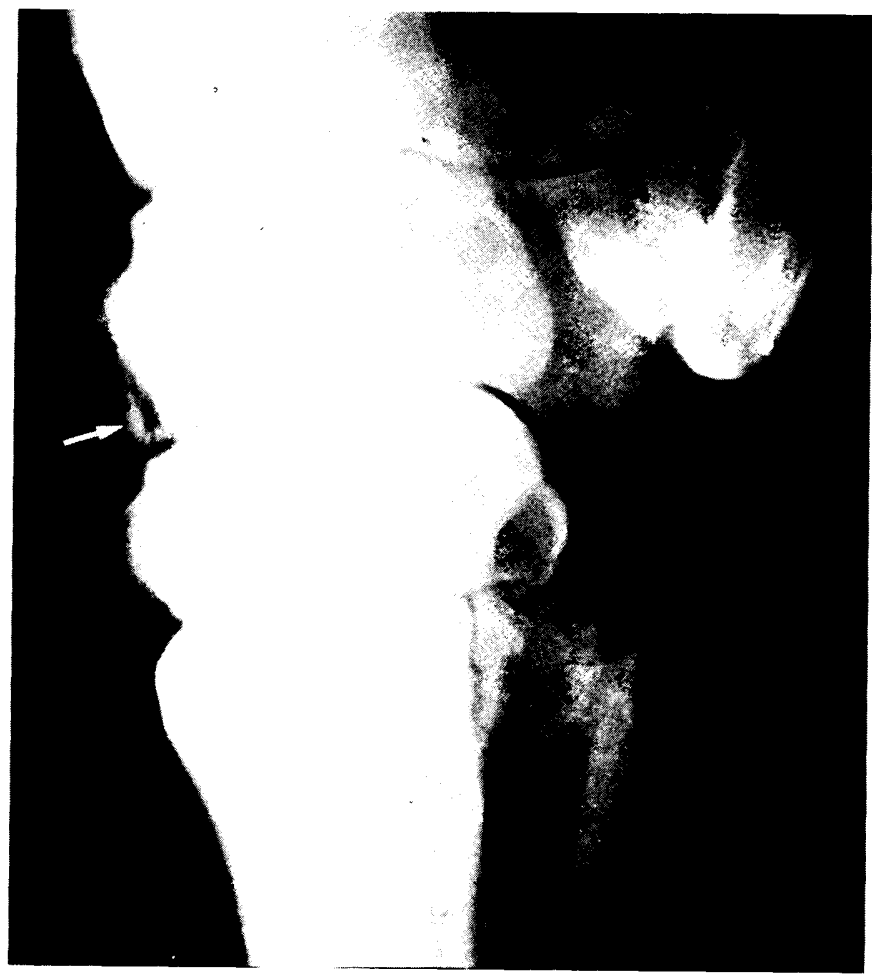

Abb. 9: Eine laterale APLMO-Aufnahme eines Dreijährigen im $30^{\circ}$-Winkel, auf der man als Chip einen frakturierten arthritisbedingten Osteophyten an der distalen kraniomedialen Fläche des Os carpi radiale sieht.

Fraktur ans Licht (Abb. 11), und der Chip wurde chirurgisch entfernt. Außerdem beseitigte man eine sehr große augenförmige „Kissing lesion“ am proximalen Rand des Os carpale tertium, die auf keinem der Röntgenbilder sichtbar war.

Abb. 12 zeigt die Röntgenaufnahme eines zweijährigen Fohlens, das akut lahm ging. Es war eine vermehrte Gelenkfüllung sowie Schmerzhaftigkeit beim Abbeugen feststellbar. Die Chip-Fraktur an der kraniolateralen Fläche
Abb. 10: Eine APLMO-Aufnahme desselben Pferds von lateral im $45^{\circ}$. Winkel, die das veränderte röntgenologische Erscheinungsbild bei einer Winkelvergrößerung des Zentralstrahls um $15^{\circ}$ zeigt.

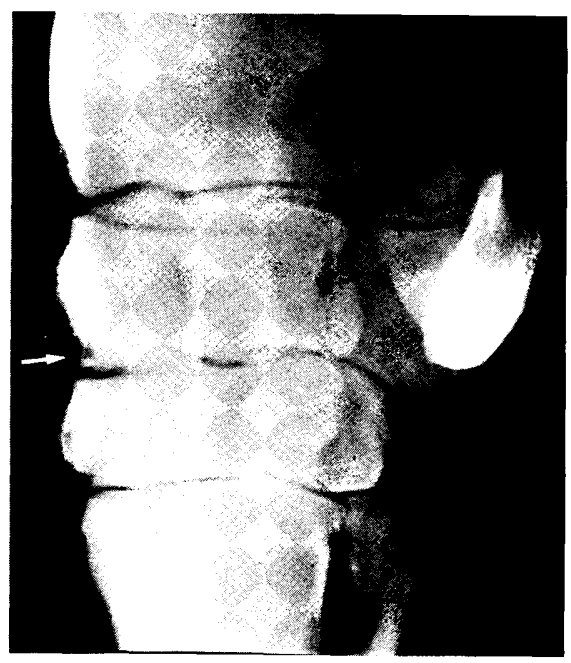

Abb. 11: APLMO-Ansicht bei einem Dreijährigen, in der eine große Chip-Fraktur zu sehen ist.

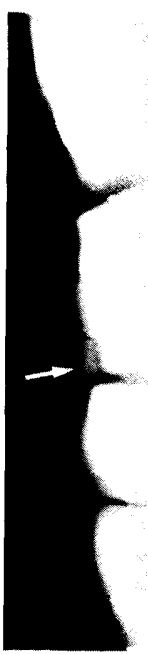

Abb. 12: APLMO-Aufnahme eines zweijährigen Pferds, auf der man eine Chip-Fraktur an der kraniolateralen Fläche des distalen Radiusendes sieht

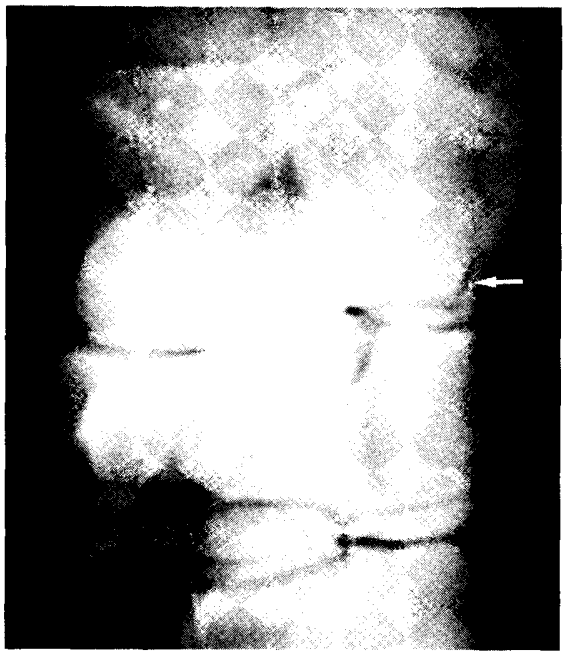


des distalen Radiusteils stellte sich auf allen Aufnahmen mit anderem Strahlengang nicht dar.

\section{Slab-Frakturen}

Park et al. (1970) fanden, daß Slab-Frakturen meist an der kranialen Oberfläche des Os carpale tertium auftreten und daß sich die Frakturlinie vom Interkarpalgelenk bis zum Gelenkspalt des Karpometakarpalgelenks erstreckt. Gewöhnlich sind die Frakturen in der Seitenansicht zu sehen; wenn der Verdacht auf eine Slab-Fraktur besteht, sind jedoch zwei den äußeren Umriß darstellende Aufnahmen (tangential) ratsam, die getrennt die proximale und die distale Reihe der Karpalgelenksknochen deutlich hervortre-

\section{Literatur}

Adams, O. R. (1974): Lameness in horses, 3rd edn. Lea \& Febiger, Philadelphia.

Gertson, K. E., und Daweson, H. A. (1976): Sagittal fracture of the third carpal bone in a horse. J. Am. vet. med. Ass. 169, 633-634.

McIllwraith, C. W., und Fessler, J. (1978): Arthroscopy in the diagnosis of equine joint disease. J. Am. vet. med. Ass. 172, 263.

Manning, J. P., und St. Clair, L. E. (1972): Carpal hyperextension and arthrosis in the horse. Am. Ass. equine Pract. 173-181.

Myers, V.S. (1965): Confusing radiologic variations at the distal end of the radius of the horse. J. Am. vet. med. Ass. 147, 1310-1312.

Park, R. D., Morgan, J. P., und O'Brien, T. (1970): Chip fractures in the carpus of the horse; a radiographic study of their incidence and location. J. Am. vet. med. Ass. 157, 1305-1312.

Smallwood, J. E., und Shivelly, M. J. (1979): Radiographic and xeroradiographic anatomy of the equine carpus. Equine Pract. 1 (1), 22-28. ten lassen. Gertson und Daweson (1976) beschrieben eine Sagittalfraktur des Os carpale tertium, welche auf den Röntgenbildern in den fünf Standardprojektionen unsichtbar und nur auf einer proximal-distalen Tangentialansicht der distalen Karpalknochenreihe zu entdecken war.

\section{Andere klinisch bedeutsame Veränderungen}

Hickman (1982; persönliche Mitteilung) sah eine Ossifikation des Radiuskopfes des oberflächlichen Zehenbeugers (oberes Unterstützungsband) in zwei Fällen, die sich röntgenologisch am kaudalen Rand des distalen Radiusabschnitts zeigte. Mit der chirurgischen Entfernung erreichte man bei beiden Pferden Heilung.

Tew, W., und Hotchkiss, $R$. (1981): Synovial fluid analysis in equine joint disorders. Equine vet. Sci. 1, 163.

Thrall, D. E., Lebel, J. L., und O'Brien, T. R. (1971): A five-year survey of the incidence and location of equine carpal chip fractures. J. Am. vet. med. Ass. 158, 1366-1368.

Den Kollegen in der Praxis und an der Equine Research Station, Newmarket, sei für die zur Verfügung gestellten Röntgenaufnahmen und die sachdienlichen Ratschläge gedankt.

\section{P. N. Burguez}

Beaufort Cottage Stables

High Street, Neremarket, Suffolk CB8 8JS, England

Erschienen in Equine Veterinary Journal (1984) 16 (3), 159-162.

Übersetzt und veröffentlicht mit freundlicher Genehmigung der British Equine Veterinary Association. 